\title{
The Clinical Significance of MIF Serum-Level as an Early Predictive Marker of Cardiovascular Risk in Patients with Vitiligo
}

\author{
Annie Riera-Leal ${ }^{1,2}$, José Alberto Tlacuilo-Parra ${ }^{3}$, Yveth Marlene Ortiz-García ${ }^{2,4}$, Pedro Gutiér- \\ rez-Fajardo $^{5}$, Alejandra Garcia-Orozco ${ }^{2,6}$, Natalia Álvarez-Abraham ${ }^{7}$, Jorge Hernández-Bello ${ }^{8}$, \\ José Francisco Muñoz-Valle ${ }^{8}$, Edsaul Emilio Perez-Guerrero ${ }^{8}$, Andrea Carolina Machado-Sul- \\ baran $^{2,6}$, Elizabeth Guevara-Gutiérrez ${ }^{7 *}$ and Lizbeth Riera-Leal ${ }^{9 *}$ \\ ${ }^{1}$ Postdoctoral degree, University of California, USA \\ ${ }^{2}$ Doctorado en Ciencias Biomédicas, Centro Universitario de Ciencias de la Salud, Universidad de Guadalajara, México \\ ${ }^{3}$ Medical Research Division, UMAE Hospital de Pediatria, México \\ ${ }^{4}$ Instituto de Investigación en Odontología, Centro Universitario de Ciencias de la Salud, Universidad de Guadalajara, México \\ ${ }^{5}$ Cardiotest Laboratorio de Ecocardiografía, Hospital Bernardette, México \\ ${ }^{6}$ Laboratorio de Inmunología, Centro Universitario de Ciencias de la Salud, Universidad de Guadalajara, México \\ ${ }^{7}$ Instituto Dermatológico de Jalisco "Dr. José Barba Rubio", Secretaría de Salud Jalisco, México \\ ${ }^{8}$ Instituto de Investigación en Ciencias Biomédica, Centro Universitario de Ciencias de la Salud, Universidad de Guadalajara, México \\ ${ }^{9}$ Hospital General Regional número 45, IMSS, México
}

*Corresponding author: Lizbeth Riera Leal, MD, Monte Albán 1351, Col. Independencia Oriente, Guadalajara, Jalisco, México.

Elizabeth Guevara Gutiérrez, MD, Monte Olimpo, Col. Independencia, Guadalajara, Jalisco, México.

*Both authors contributed equally to this work.

To Cite This Article: Annie Riera-Leal, José Alberto Tlacuilo-Parra, Yveth Marlene Ortiz-García. The Clinical Significance of MIF Serum-Level as an Early Predictive Marker of Cardiovascular Risk in Patients with Vitiligo. Am J Biomed Sci \& Res. 2021 - 12(4). AJBSR.MS.ID.001775. DOI: 10.34297/AJBSR.2021.12.001775.

Received: 紫 December 16, 2020; Published: 眥 April 22, 2021

\begin{abstract}
Background: In the pathogenesis of vitiligo, pro-inflammatory cytokines have been linked to insulin resistance, dyslipidemia, and atherosclerosis; which are associated with cardiovascular diseases (CD). Macrophage Migration Inhibitory Factor (MIF) is a pro-inflammatory cytokine that is welldescribed in the pathogenesis of several autoimmune disorders. Also, MIF has been involved in the development of atherosclerosis and has emerged as a cardiovascular risk (CR) factor.
\end{abstract}

Objective: Our objective was to analyze the relationship between MIF and CR in patients with vitiligo.

Method: We evaluated dyslipidemia, insulin resistance, and CR according to the Castelli Atherogenic Index, ultrasensitive C-reactive protein (usCRP), and carotid atherosclerosis by the intima-media thickness (IMT) in 50 patients and 50 healthy subjects. MIF concentrations were measured by ELISA assay. MIF tissue expression was evaluated by automated immunohistochemistry. The relationship of these cardiovascular alterations was investigated concerning the extent and activity of vitiligo. Results A higher prevalence of low HDL cholesterol (HDL-c) and moderate/high CR according to Castelli Atherogenic Index was observed in the vitiligo group. Subjects with generalized/universal vitiligo had more CR as stated in higher usCRP values, Castelli Index, Carotid IMT averages, and lower HDL-c levels. MIF serum levels were higher in patients with active disease, HOMA-IR $>2.4, \mathrm{HDL}<40 \mathrm{mg} / \mathrm{dL}$, and high-risk usCRP. Serum MIF levels were negatively correlated with variables of CR in vitiligo. It was increased MIF expression in depigmented skin. Conclusions This study provides the first evidence for the involvement of MIF in the evolution of CR in vitiligo.

Keywords: MIF; Cardiovascular risk; Vitiligo 


\section{Introduction}

Vitiligo is a common dermatologic condition characterized by a loss of melanocytes [1]. The etiology and the exact physiopathological mechanism of this disease are unknown; however, a melanocyte death mediated by the immune system has been suggested [2]. Added to this, antibody-mediated, cell-mediated, and cytokinemediated mechanisms have all accumulated supportive evidence that the damage to the homeostasis of melanocytes is caused by autoimmune processes $[3,4]$.

High levels of tumor necrosis factor-alpha (TNF- $\alpha$ ), interleukin-1 alpha (IL-1 $\alpha$ ), and interleukin-6 (IL-6) have been observed in the lesions of vitiligo patients; [5]. Similarly, high granulocyte-macrophage colony-stimulating factors have been found in the serum [6]. These cytokines have also been related to the development of insulin resistance and dyslipidemia that lead to vascular damage and atherosclerosis $[7,8]$. Thus, it is possible that patients with vitiligo would have an increased risk of cardiovascular diseases (CDs).

The common denominator of most CDs is a chronic inflammatory pathology of the vessel wall called atherosclerosis [9]. The atherogenesis process development involves specific cellular and molecular responses that culminate into an acute event due to plaque erosion or rupture. The primary defect occurs at the vascular endothelial cells which become dysfunctional, develop pores in the junctions, and provoke inflammatory cells recruitment to the subendothelial region. A chronic inflammatory-fibroproliferative response characterizes the subsequent growth of the plaque [10] The movement of monocytes and T cells from the bloodstream to the damaged vascular wall, together with the platelets, perpetuates the inflammatory environment through the secretion of several vasoactive chemokines and cytokines [11]. There is compelling evidence about the role of immunity in atherosclerosis, and CDs are the primary cause of death among patients with various autoimmune diseases like lupus and rheumatoid arthritis [10].

The Macrophage Migration Inhibitory Factor (MIF) is an inflammatory cytokine with an important role in apoptosis. This cytokine has a well-established role in the onset and development of several immune-inflammatory diseases [12-15] but it has been poorly studied in dermatological disorders [16]. The powerful effects of MIF on autoimmune dermatological illness include the activation and differentiation of Th1 and Th17 cells, which are characterized by producing the major pro-inflammatory cytokines involved in its pathogenesis, such as IL-1, IL-6, IFN- $\gamma$, TNF- $\alpha$, and IL$17[17,18]$. In recent years, MIF has also been broadly implicated in the atherogenesis process [19] as it can regulate the inflammatory and immune signaling in atherosclerosis, potentiate neointimal thickening by promoting the monocyte and $\mathrm{T}$ cell recruitment and it induces proliferation of the smooth muscle cells in vessels [2022].
Several research groups have shown that high MIF serum levels might be a novel biomarker for the risk of cardiovascular disease [23-25]. In this study, we aimed to assess the potential of serum MIF as an early predictive marker of atherosclerosis and cardiovascular risk (CR) in patients with vitiligo.

\section{Material and Methods}

\section{Patients with vitiligo and control subjects}

A cross-sectional study was carried out in the Dermatology Institute of Jalisco "Dr. José Barba Rubio" after authorization from the ethics committee (No.17-015) of the institution and the signing of informed consents from all participants. Our study enrolled 50 patients with vitiligo and 50 subjects with no medical record of vitiligo, autoimmune or chronic disease, named as Control Subjects (CS), matched by age, gender, and body mass index (BMI). All individuals were of Mexican origin and at least 18 years of age. Subjects with a history of a congenital or acquired condition which during its evolution had metabolic disorders and atherosclerosis; those who during the physical examination had findings compatible with diseases associated with metabolic, lipid disorders, or atherosclerosis, and those with a current consumption of hypoglycemic, lipid-lowering medications, oral steroids or other systematic pharmacological treatment, and pregnant women and smokers, were excluded from the study.

\section{Clinical measurements}

The extent of the vitiligo was classified into localized (focal, segmental, and mucosal), generalized (acro-facial, vulgar, and mixed), and universal [6]. Disease activity was measured with the Vitiligo Activity Index, which is based on the presence of new lesions and/or increases in the size of pre-existing injuries during a period from 6 weeks to 1 year, with a score from -1 to +4 where the higher score indicates the greater activity [7]. A score of +1 to +4 was considered as active vitiligo, while inactive vitiligo with scores of -1 and zero. BMI was calculated by dividing weight (in kilograms) by height (meters squared) $(\mathrm{kg} / \mathrm{m} 2)$.

A 12-h fasting peripheral blood sample was obtained. Insulin was measured with electrochemiluminescence and insulin resistance was estimated using the Homeostasis Model Assessment (HOMAIR), which was calculated using the following formula: glucose (mg/ $\mathrm{dL}) \mathrm{x}$ insulin $(\mu \mathrm{U} / \mathrm{mL})$ by 405 ; [26-28] values $>2.4$ were considered as resistance to insulin [29]. Serum lipids were measured with the enzymatic colorimetric method. Total cholesterol (TC) $\geq 200 \mathrm{mg} /$ dL, low-density lipoprotein (LDL) $\geq 130 \mathrm{mg} / \mathrm{dL}$, triglycerides (TG) $\geq 150 \mathrm{mg} / \mathrm{dL}$ and High-Density Lipoprotein (HDL) < $40 \mathrm{mg} / \mathrm{dL}$ were considered to be abnormal [30].

The CR was determined with the Castelli Atherogenic Index, which was calculated by dividing the TC (mg/dL) by the HDL (mg/ $\mathrm{dL}$ ) and was classified as low risk ( $<5$ in males, $<4.5$ in females), moderate risk (5-9 in males, 4.5-7 in females), and high risk ( $>9$ in 
males, > 7 in females) (30). Ultrasensitive C-reactive protein (usCRP) was measured using the turbidimetric method. CR was classified as low (<1 mg/L), medium (1-3 mg/L), and high (>3 mg/L) [31-33].

Carotid atherosclerosis was evaluated using the intima-media thickness (IMT) defined as the measurement of the mean thickness over the distal $1 \mathrm{~cm}$ of the wall of the left and right common carotid arteries length. The measurements were done in triplicate, and the values obtained were averaged to obtain the IMT $[34,35]$. The measurement was semiautomatic using B-mode ultrasound (Philips iE33) with a transducer (L11-3).

\section{MIF Serum-level quantification}

Serum was obtained from all individuals at the time of study inclusion; MIF serum levels were quantified by a commercial Enzyme-Linked ImmunoSorbent Assay (ELISA) kit (LEGEND MAX® Human Active MIF ELISA Kit; BioLegend®, San Diego, CA, USA) according to the manufacturers, instructions. The sensitivity of the assay was $17.4+9.2 \mathrm{pg} / \mathrm{mL}$.

\section{MIF Expression in situ by automated immunohistochem- istry}

A total of 20 specimens of depigmented and perilesional skin from vitiligo patients were examined. Besides, ten samples of healthy skin tissue from the surface of patients who underwent dermatological surgery for the removal of benign lesions were incorporated in the analysis. subjects included signed the written informed consent. Two experienced dermatopathologists examined and classified the samples.

Serial sections $(5 \mu \mathrm{m})$ from the formalin-fixed paraffinembedded blocks were used for the detection of MIF protein using immunohistochemistry. The MIF primary antibody (Cat. No. sc20121 RRID: AB_648587) was used at a concentration of 1:50 in $100 \mu \mathrm{l}$ of a $1 \mathrm{X}$ concentrated solution of Tris-buffered saline with $0.1 \%$ Tween-20 (TBS-T). Slides were loaded into the BenchMark ULTRA instrument (Cat. no. N750-BMKU-FS 05342716001; Ventana
Medical Systems, Inc.). Ventana Medical Systems, Inc provided benchmark ULTRA reagents, solutions, kits, and accessories. The OptiView Amplification kit was used in conjunction with the OptiView DAB IHC Detection kit to increase the staining intensity. Working conditions included: incubation with the MIF primary antibody, 28 minutes; hematoxylin stain, 8 minutes; and bluing reagent, 4 minutes. The entire process lasted approximately 2 hours. Ventana Medical Systems, Inc supplied the mouse secondary antibody. The default temperature was $95^{\circ} \mathrm{C}$. As a negative control of the process in some samples, the primary antibody was omitted.

An optical microscope (Carl Zeiss AG, Oberkochen, Germany) coupled to a digital camera Coolsnap (Photometrics, Tucson, USA) was used to obtain the histopathological images of the complete sample. Depending on the extension of the tissue five to ten fields were analyzed. For the digital analysis, the cell counter function of the Image-pro Plus 6.0® software (Media Cybernetics, Inc., Rockville, MD, USA) was employed following the instructions of the program. The optical densities corresponding to the intensity of the brown color were calculated.

\section{Statistical analysis}

The obtained data were analyzed with the statistical software SPSS v. 23 (IBM Corp., Armonk, NY, USA) and GraphPad Prism v. 7 (GraphPad Software, Inc., La Jolla, CA, USA). Mean and standard deviations were obtained. For comparison between proportions, chi-square (X2) or Fisher exact tests were used. For continuous variables analysis, the Student-t or Mann-Whitney U tests were used, according to their normal distribution. For the comparison of more than two groups, a one-way analysis of variance (ANOVA) with Bonferroni correction was employed. As a measure of association, the odds ratio (OR) was determined with a $95 \%$ confidence interval (95\% CI). The Spearman linear correlation analysis was performed to assess the association of MIF serum levels with the representative CR variables evaluated in the study. A p-value $\leq 0.05$ was considered statistically significant.

\section{Results}

\section{Description of the clinical and demographic characteristics of the study subjects}

Table 1: Demographic and clinical characteristics of patients with vitiligo and control subjects.

\begin{tabular}{|c|c|c|c|}
\hline Variables & Vitiligo & Control Subjects & p-value \\
\hline \multicolumn{4}{|c|}{ Demographic and clinic characteristics } \\
\hline Age (years) ${ }^{a}$ & $45 \pm 13$ & $45 \pm 13$ & $0.9^{*}$ \\
\hline \multicolumn{4}{|l|}{$\operatorname{Sex}^{b}$} \\
\hline Male (\%) & $19(38)$ & $19(38)$ & $1.0^{* *}$ \\
\hline Female (\%) & $31(62)$ & $31(62)$ & $1.0^{* *}$ \\
\hline Body Mass Index ${ }^{a}$ & $28 \pm 6$ & $28 \pm 5$ & $0.6^{*}$ \\
\hline Family history of vitiligo $\mathrm{n}(\%)^{\mathrm{b}}$ & $25(50)$ & $4(8)$ & $0.0001^{* *}$ \\
\hline \multicolumn{4}{|c|}{ Disease status } \\
\hline Duration of disease (years) $^{c}$ & $13(1-59)$ & - & - \\
\hline Disease activity (VIDA score) ${ }^{c}$ & $2(-1-+4)$ & - & - \\
\hline
\end{tabular}




\begin{tabular}{|c|c|c|c|}
\hline${\text { Classification of vitiligo } \mathrm{n}(\%)^{\mathrm{b}}}^{\mathrm{b}}$ & & - & - \\
\hline Generalized & $42(84)$ & - & - \\
\hline Localized & $7(14)$ & - & - \\
\hline Universal & $1(2)$ & - & \\
\hline
\end{tabular}

a: Data provided in mean \pm SD, b: Data presented in $n$ and percentage, c: Data presented in median (p5-p95)

CS: Control Subject; VIDA: Vitiligo Disease Activity Index. *: p-value was calculated by the t-Student test; **: p-value was calculated by $\square \square$-test.

In both study groups, there was a predominance of females (62\%), and the average age was $45 \pm 13$ years (range 18-74 years). The average BMI in the vitiligo group was $28 \pm 6$ (range 18-43) and $28 \pm 5$ (range 20-38) for the CS. Vitiligo was generalized in 42 subjects (84\%), localized in seven (14\%), and universal in one (2\%). 34 cases (68\%) had active vitiligo and 16 cases $(32 \%)$ were classified as stable. The patients had a median disease evolution time of $13 \pm 13$ years (range, 1-59 years). Concerning the genetic component, it was observed that $50 \%$ of patients had a family history of the disease, whereas, in CS, it was $8 \%$ (OR 11.5; 95\% CI, 3.6 - 36.7; $\mathrm{p}<0.0001$ ) (Table 1).

\section{High serum levels of MIF are related to the degree of activity of vitiligo}

A)

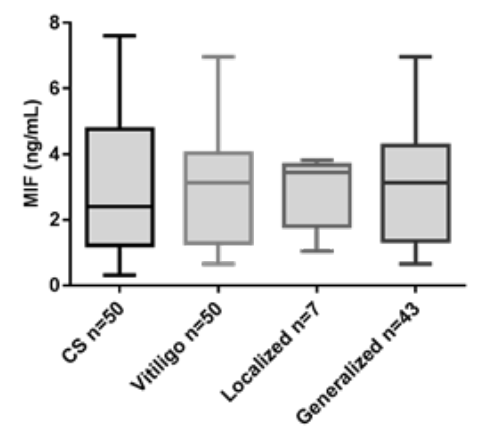

C)

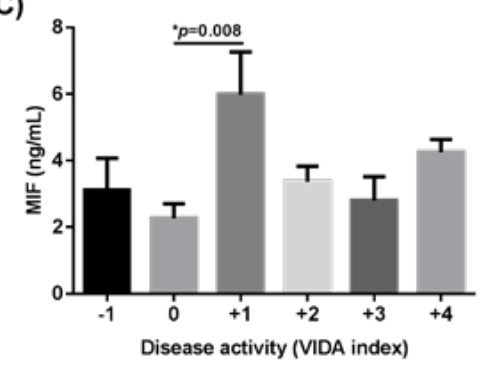

B)

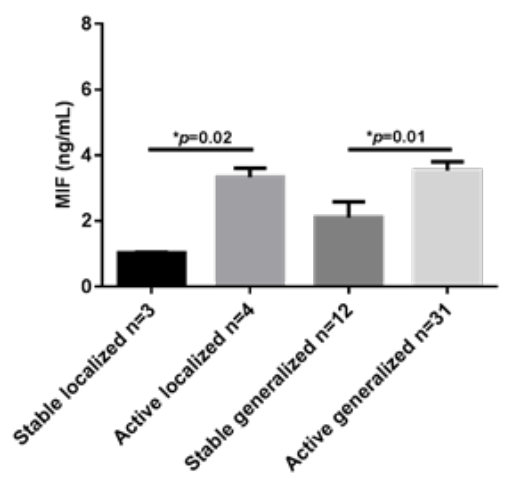

Figure 1: Comparison of MIF concentrations between study groups (A). Levels of MIF in patients with vitiligo are classified according to the degree of activity of the disease $(B)$ and according to the VIDA scale $(C)$. Data are expressed as mean \pm standard error. Statistical analysis was performed using one-way ANOVA. MIF: macrophage migration inhibitory factor; ng: nanogram; mL: milliliters; CS: control subjects; VIDA: Vitiligo Disease Activity Score. *: statistically, significant differences were considered with a $p$-value $<0.05$.

The concentration of MIF serum levels was higher in patients with vitiligo than in CS (3.1 ng/mL vs. $2.6 \mathrm{ng} / \mathrm{mL}$, respectively), but this did not reach statistical significance $(p=0.7)$ (Figure $1 \mathrm{~A})$. However, when the group with vitiligo was subclassified according to the degree of activity, a significant difference was observed between active versus stable patients, regardless of the disease extent $(\mathrm{p}=0.02$ for localized vitiligo, and $\mathrm{p}=0.01$ for generalized vitiligo) (Figure 1B). Moreover, patients with a disease activity index score of +1 had the highest levels in comparison with the other scores $(-1,0,+2,+3,+4)(p=0.008$, Figure $1 C)$.

\section{Insulin resistance and dyslipidemia in patients with vitiligo}

Insulin resistance was evident in 25 patients (50\%) and in 18 (36\%) CS, $\mathrm{p}=0.15$. Among 25 patients with vitiligo and insulin resistance, 22 (88\%) had generalized/universal vitiligo and three $(12 \%)$ had localized vitiligo $(\mathrm{p}=0.50)$. Active disease was reported in 16 cases $(64 \%)$ and stable in nine cases $(36 \%, p=0.54)$ (data are not shown). HDL decrease was more frequent in the vitiligo patients in comparison with the CS [40\% vs. $20 \%$, respectively; $\mathrm{p}=0.02, \mathrm{OR}=3,95 \% \mathrm{CI}: 1-7]$. The $100 \%$ of patients with HDL $<40$ 
$\mathrm{mg} / \mathrm{dL}$ presented generalized/universal vitiligo. Alterations in the remainder of the lipids showed no significant difference between groups (Table 2).
HOMA-IR index was similar between vitiligo patients and CS [2.9 \pm 2 (range $0.3-8.9$ ) vs. $2.3 \pm 1.7$ (range 0.5-7.4, respectively, $\mathrm{p}=0.17$ )], and among vitiligo groups (Figure $2 \mathrm{~A}$ ).

Table 2: Presence of dyslipidemia in subjects with and without vitiligo.

\begin{tabular}{|c|c|c|c|c|}
\hline \multirow{2}{*}{ Variable } & Vtiligo & Control Subjects & \multirow{2}{*}{$\mathbf{p}$} & OR \\
\hline & $n=50(\%)$ & $\mathrm{n}=\mathbf{5 0}(\%)$ & & $(95 \% \mathrm{CI})$ \\
\hline $\mathrm{TC}>200 \mathrm{mg} / \mathrm{Dl}$ & $14(28)$ & $20(40)$ & 0.2 & - \\
\hline $\mathrm{LDL}>130 \mathrm{mg} / \mathrm{dL}$ & $10(20)$ & $15(30)$ & 0.24 & - \\
\hline $\mathrm{HDL}<40 \mathrm{mg} / \mathrm{dL}$ & $20(40)$ & $10(20)$ & $* 0.02$ & $3(1-7)$ \\
\hline $\mathrm{TG}>150 \mathrm{mg} / \mathrm{dL}$ & $23(46)$ & $18(36)$ & 0.3 & - \\
\hline
\end{tabular}

TC: Total Cholesterol; LDL: Low-Density Lipoprotein; HDL: High-Density Lipoprotein; TG: Triglycerides * $p<0.05 \square 2$

A)

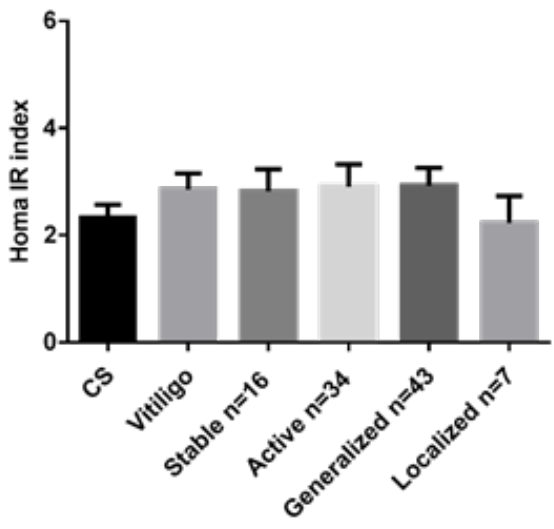

B)

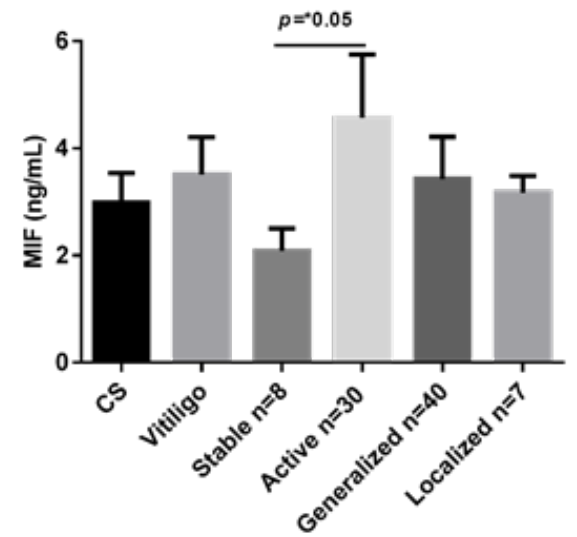

Homa IR index $>2.4$

Figure 2: HOMAR IR index in the study groups (A). MIF concentrations in patients with HOMAR IR greater than 2.4 (B). Data are expressed as mean \pm standard error. Statistical analysis was performed using $U$ the Mann-Whitney. MIF: macrophage migration inhibitory factor; ng: nanogram; $\mathrm{mL}$ : milliliters; CS: control subjects; *: statistically significant differences were considered with a $p$-value $<0.05$.

Patients with HOMA-IR $>2.4$ or HDL $<40 \mathrm{mg} / \mathrm{dL}$ have higher serum levels of MIF

\section{MIF (ng/mL) and HDL $<40 \mathrm{mg} / \mathrm{dL}$}

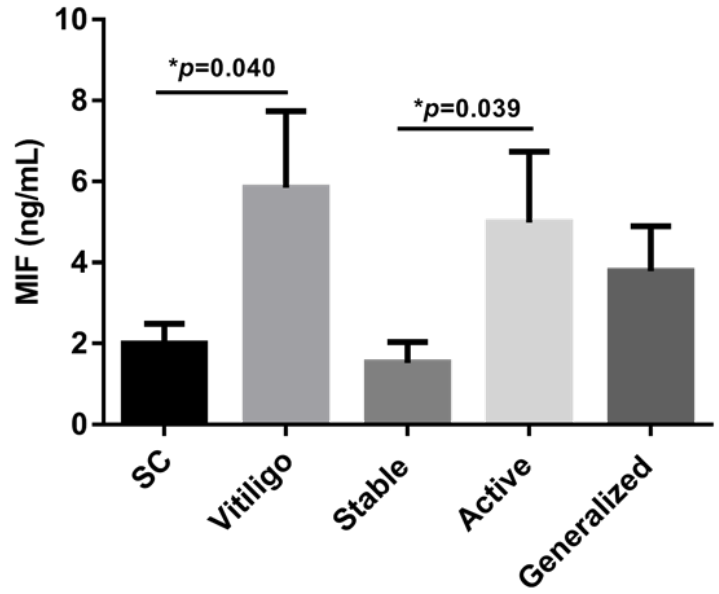

Figure 3: MIF levels in patients with dyslipidemia. Data are expressed as mean \pm standard error. Statistical analysis was performed using oneway ANOVA. HDL: High-density lipoprotein; MIF: macrophage migration inhibitory factor; ng: nanogram; mL: milliliters; CS: control subjects; $\square$ : statistically significant differences were considered with a $p$-value $<0.05$. 
Patients with active vitiligo and resistance to the insulin (HOMA-IR >2.4) showed higher levels of MIF than those stable ones (mean 3.061 vs. 1.875; p=0.05) (Figure 2B). Serum levels of MIF were also significantly higher in vitiligo patients with $\mathrm{HDL}<40 \mathrm{mg} /$ $\mathrm{dL}$ (mean 3.2 vs. 1.7; $\mathrm{p}=0.04$ ), especially in those with active vitiligo (mean 3.5 vs. $1.0 ; \mathrm{p}=0.039$ ) (Figure 3 ).

\section{Patients with vitiligo have a higher Atherogenic Index of Castelli}

The average of the Castelli Atherogenic Index was higher in the vitiligo group $[4.53 \pm 1.4 \mathrm{mg} / \mathrm{L}$ (range $2-8.9$ ) vs. $3.91 \pm 1.2$ $\mathrm{mg} / \mathrm{L}$ (range 1.8-7.8), respectively] than in the CS $(\mathrm{p}=0.02)$. This atherogenic index was also higher in subjects with generalized/ universal vitiligo than in those with localized vitiligo (4.67 vs. 3.67 , respectively, $\mathrm{p}=0.01$ ). There was no relationship between the average of the Castelli Index with the disease activity (active vitiligo 4.42 vs. stable vitiligo 4.61, $\mathrm{p}=0.31$ ) (Figure $4 \mathrm{~A}$ ).

Moderate/high CR was present in $40 \%$ of subjects with vitiligo and in $22 \%$ of CS ( $p=0.05,0 R=2,95 \%$ CI: 0.91-6) (data not shown). There were no significant differences in the MIF serum levels among vitiligo groups according to CR (moderate/high or low) assessed by the Castelli Index (Figure 4B \& 4C).
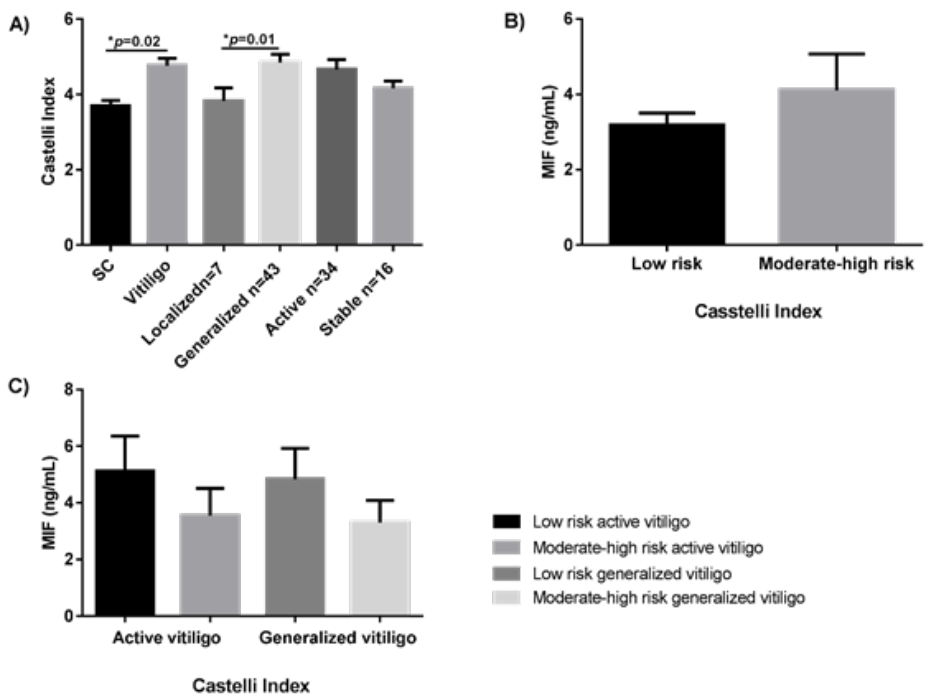

Castelli Index

Figure 4: Atherogenic Index of Castelli comparison among study groups (A). MIF serum levels in patients with vitiligo according to CR moderate/ high or low (B), and among vitiligo groups according to Castelli Index (C). Data are expressed as mean \pm standard error. Statistical analysis was performed using one-way ANOVA and U the Mann-Whitney. MIF: macrophage migration inhibitory factor; ng: nanogram; mL: milliliters; CS: control subjects; *: statistically significant differences were considered with a p-value $<0.05$.
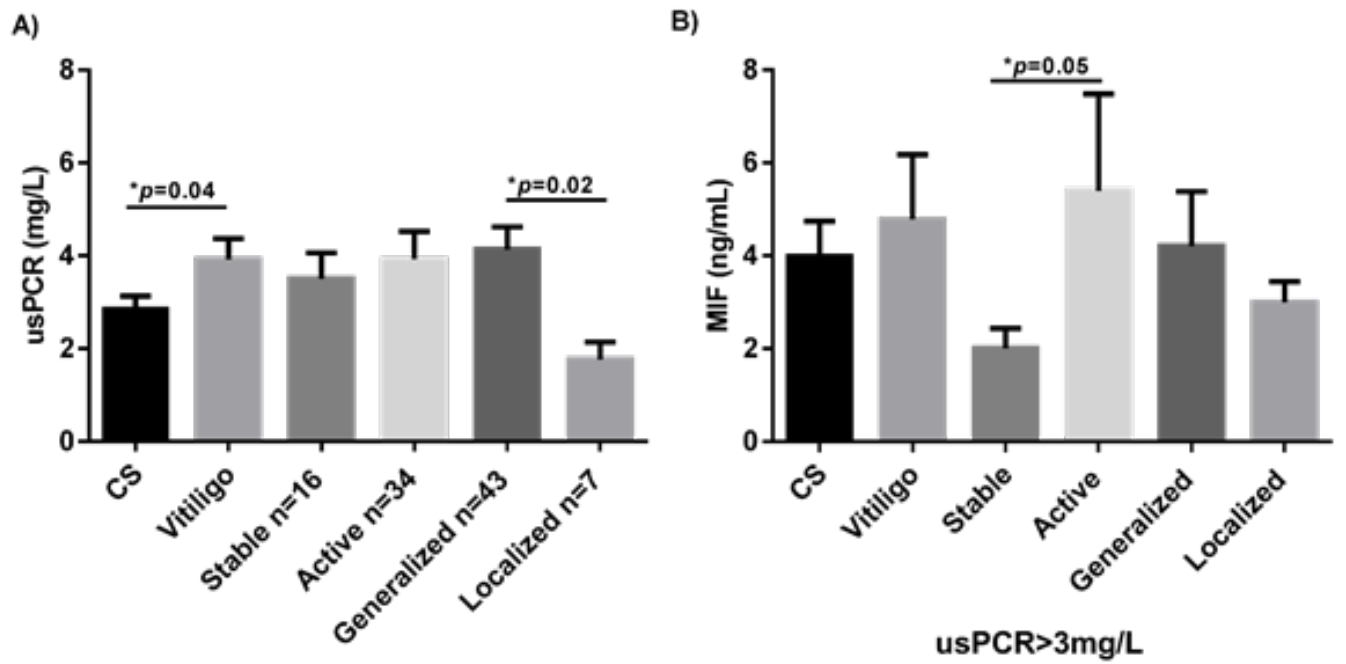

Figure 5: usPCR levels between study groups (A). MIF levels between study groups of subjects with usPCR<3mg/L (B). Data are expressed as mean \pm standard error. Statistical analysis was performed using $U$ the Mann-Whitney. MIF: macrophage migration inhibitory factor; ng: nanogram $\mathrm{mL}$ : milliliters; CS: control subjects; usPCR: Ultra-Sensitive C-Reactive Protein. *: statistically, significant differences were considered with a p-value $<0.05$. 


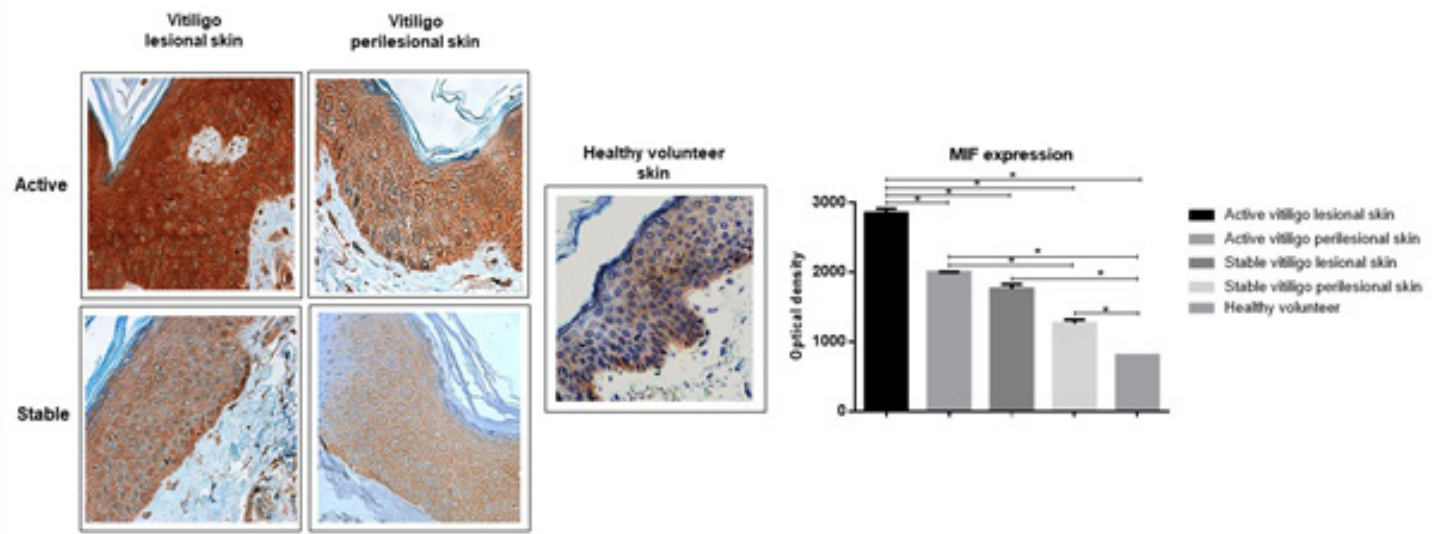

Figure 6:

The average of the usCRP was higher in the patients with vitiligo vs. the CS group (3.925 \pm 0.45 vs.2.841 $\pm 0.29, \mathrm{p}=0.04)$. Patients with generalized vitiligo had higher levels of usCRP than patients with the localized variant of the disease (medians, 2.800 vs. 1.750 , respectively; $\mathrm{p}=0.02$ ) (Figure $5 \mathrm{~A}$ ); however, there were no significant differences between patients with vitiligo vs. the CS group concerning the degree of atherogenic or CR (Table 3). Among 21 patients with high-risk levels of usCRP, 19 (90\%) had generalized/universal vitiligo and two (10\%) had localized vitiligo ( $\mathrm{p}=0.03) ; 12$ (57\%) had active vitiligo and nine (43\%) had stable disease $(\mathrm{p}=0.16)$ (data not shown).

Table 3: Cardiovascular risk according to usPCR between subjects with and without vitiligo.

\begin{tabular}{|c|c|c|c|}
\hline \multirow{2}{*}{ Atherogenic risk } & Vitiligo & Control subjects & \multirow{2}{*}{ p value } \\
\cline { 2 - 3 } & $\mathbf{n = 5 0}(\mathbf{5 0})$ & $\mathbf{n = 5 0}(\mathbf{5 0})$ & \\
\hline Low $(<1 \mathrm{mg} / \mathrm{L})$ & $2(4)$ & $1(2)$ & $0.50^{*}$ \\
\hline Medium $(1-3 \mathrm{mg} / \mathrm{L})$ & $27(54)$ & $33(66)$ & $0.22^{* *}$ \\
\hline High $(>3 \mathrm{mg} / \mathrm{L})$ & $21(42)$ & $16(32)$ & $0.30^{* *}$ \\
\hline
\end{tabular}

${ }^{* *} \mathrm{p} \square 2$

usCRP, ultrasensitive C-reactive protein

\section{Patients with generalized/universal vitiligo have a higher Carotid IMT average \\ IMT: Intima-Media Thickness}

Table 5: Relation of carotid IMT with the clinical characteristics of vitiligo.

\begin{tabular}{|c|c|c|c|}
\hline \multirow{2}{*}{ Variable } & Localized Vitiligo & Generalized/Universal Vitiligo & \multirow{2}{*}{ p Value } \\
\cline { 2 - 4 } & $\mathbf{n = 7}$ (average in $\mathbf{~ m m}$ ) & $\mathbf{n}=\mathbf{4 3}$ (average in $\mathbf{~ m m}$ ) & 0.14 \\
\hline Right common carotid & 0.48 & 0.52 & $* 0.04$ \\
\hline Right bulb & 0.53 & 0.59 & $* 0.02$ \\
\hline Left common carotid & 0.48 & 0.54 & $* 0.02$ \\
\hline
\end{tabular}

\section{Patients with active vitiligo and high CR/usCRP have higher serum levels of MIF}

Serum levels of MIF were evaluated in those individuals with CR evaluated through the usCRP (sCRP $>3 \mathrm{mg} / \mathrm{L}$ ). Significantly higher MIF serum levels were observed in patients with active vitiligo in comparison with the stable vitiligo patients (means: $3.259 \mathrm{ng} / \mathrm{mL}$ vs. $1.777 \mathrm{ng} / \mathrm{mL}$; $\mathrm{p}=0.05$ ) (Figure $5 \mathrm{~B}$ ).

Table 4: Carotid IMT in subjects with and without vitiligo.

\begin{tabular}{|c|c|c|c|}
\hline Variable & Vitiligo & Control subjects & \multirow{2}{*}{ p value* } \\
\cline { 2 - 3 } & $\begin{array}{c}\mathrm{n}=50 \text { (averages } \\
\text { in mm) }\end{array}$ & $\begin{array}{c}\mathrm{n}=50 \text { (averages in } \\
\mathrm{mm} \text { ) }\end{array}$ & 0.34 \\
\hline $\begin{array}{c}\text { Right common } \\
\text { carotid }\end{array}$ & 0.99 & 0.54 & 0.96 \\
\hline Right bulb & 0.59 & 0.58 & 0.35 \\
\hline Right carotid-bulb & 0.79 & 0.56 & 0.51 \\
\hline $\begin{array}{c}\text { Left common } \\
\text { carotid }\end{array}$ & 0.53 & 0.54 & 0.87 \\
\hline Left bulb & 0.6 & 0.6 & 0.74 \\
\hline Left carotid-bulb & 0.57 & 0.57 & \\
\hline
\end{tabular}

${ }^{*} p<0.05$ Student $t$-test 


\begin{tabular}{|c|c|c|c|}
\hline Variable & $\begin{array}{c}\text { Stable Vitiligo } \mathbf{n = 1 6} \text { (average in } \\
\mathbf{m m} \text { ) }\end{array}$ & $\begin{array}{c}\text { Active Vitiligo } \mathbf{n}=\mathbf{3 4} \text { (average in } \\
\mathbf{m m} \text { ) }\end{array}$ & p Value \\
\hline Right common carotid & 0.56 & 0.5 & 0.06 \\
\hline Right bulb & 0.62 & 0.57 & 0.18 \\
\hline Left common carotid & 0.55 & 0.52 & 0.19 \\
\hline Left bulb & 0.63 & 0.58 & 0.22 \\
\hline
\end{tabular}

${ }^{*} \mathrm{p}<0.05$ Student t-test

IMT: Intima-Media Thickness

Carotid IMT average between groups showed no significant differences (Table 4). Average IMT of the left carotid artery, right bulb, and left bulb was statistically higher in the group with generalized/universal vitiligo than in the group with localized

vitiligo ( $\mathrm{p}=0.04, \mathrm{p}=0.02$, and $\mathrm{p}=0.02$ for the right bulb, left common carotid, and left bulb; respectively). We found no statistically significant differences concerning disease activity (Table 5).

\section{Serum MIF values are negatively correlated with variables of CR in patients with vitiligo}

Table 6: Coefficients of linear correlation between serum levels of MIF and the cardiovascular risk variables studied in patients with vitiligo.

\begin{tabular}{|c|c|c|}
\hline Cardiovascular Risk Variables & Spearman r & p value* \\
\hline Glucose & -0.1831 & 0.2031 \\
\hline Insulin & -0.3511 & $* 0.0124$ \\
\hline HOMA-IR & -0.3055 & $* 0.0310$ \\
\hline usPCR & -0.3401 & $* 0.0157$ \\
\hline Total cholesterol & 0.03143 & 0.8285 \\
\hline LDL-C & 0.1197 & 0.4078 \\
\hline HDL-C & 0.2334 & 0.1029 \\
\hline VLDL-C & -0.3114 & $* 0.0277$ \\
\hline TG & -0.3168 & $* 0.0250$ \\
\hline HOMA-B & -0.2622 & 0.0659 \\
\hline Castelli index & -0.1411 & 0.3284 \\
\hline Right common carotid IMT & -0.1895 & 0.1876 \\
\hline Right bulb IMT & -0.2529 & 0.0764 \\
\hline Left common carotid IMT & -0.2882 & 0.0424 \\
\hline Left bulb IMT & -0.3294 & $* 0.0195$ \\
\hline
\end{tabular}

\section{${ }^{*} p<0.05$ Spearman correlation}

IMT: Intima-Media Thickness; usPCR: Ultra-Sensitive C-Reactive Protein; LDL-C: Low-Density Lipoprotein; HDL-C: High-Density Lipoprotein; VLDL-C: Very Low-Density Lipoprotein; TG: Triglycerides

Spearman's simple linear correlation between the serum level of MIF values and CR variables showed that MIF serum levels negatively correlated with insulin levels, HOMA-IR index, usCRP, VLDL-C, TG, left common carotid IMT, and left bulb IMT (Table 1). However, subsequent multivariate analysis showed only a significant correlation between MIF serum levels with the insulin serum levels and the left bulb IMT ( $p=0.03$, and $p=0.04$; respectively) (Table 6).

\section{High levels of MIF in the patches of depigmented skin of vitiligo patients}

The analysis of the optical densities for the brown staining showed that the vitiligo lesioned skin had more MIF protein expression than the perilesional skin in active vitiligo (mean difference of 847.9, optical density; $\mathrm{p}=0.006$ ). Also, patients with active vitiligo exhibit higher MIF expression than those stables ( $p=0.006$ for the lesioned skin, and $\mathrm{p}=0.005$ for the perilesional skin). All the analyses performed showed that patients with vitiligo have more skin MIF expression in comparison with the healthy volunteers (Figure 6).

\section{Molecular common pathways between vitiligo, MIF, atherosclerosis, and the cardiovascular risk}

Figure 6 highlight the molecular mechanisms involved in vitiligo that clarify the association of this pathology with MIF and the CR. Under the influence of well-known risk factors, endothelial cells become dysfunctional and this is the first step of atherosclerosis. Inflammatory processes are also key contributors to the pathogenesis of atherosclerosis. Vitiligo is an autoimmune, inflammatory disease. Activated leukocytes in vitiligo release MIF. Due to its functions, MIF could participate in the formation of the atherosclerotic plaque, by promoting cellular infiltration of monocytes/macrophages and T cells [36], through overexpression of adhesion molecules in endothelial cells and infiltrated cells [37]. It also directs migration, since MIF exhibits chemokine- 
like activities through interactions with the chemokine receptors CXCR2 and CXCR4 [19,36,37]. Furthermore, MIF regulates smooth muscle cell migration and proliferation and enhanced degradation of extracellular matrix proteins by overexpression of MMP [19]. On the other hand, it has been described that MIF can have a cardioprotective effect by activating AMPK that promotes cellular glucose uptake by inducing the trafficking of GLUT4-containing membrane vesicles to the membrane, inhibits pro-apoptotic cascades, and enhances pro-survival signaling [38] (Figure 7).

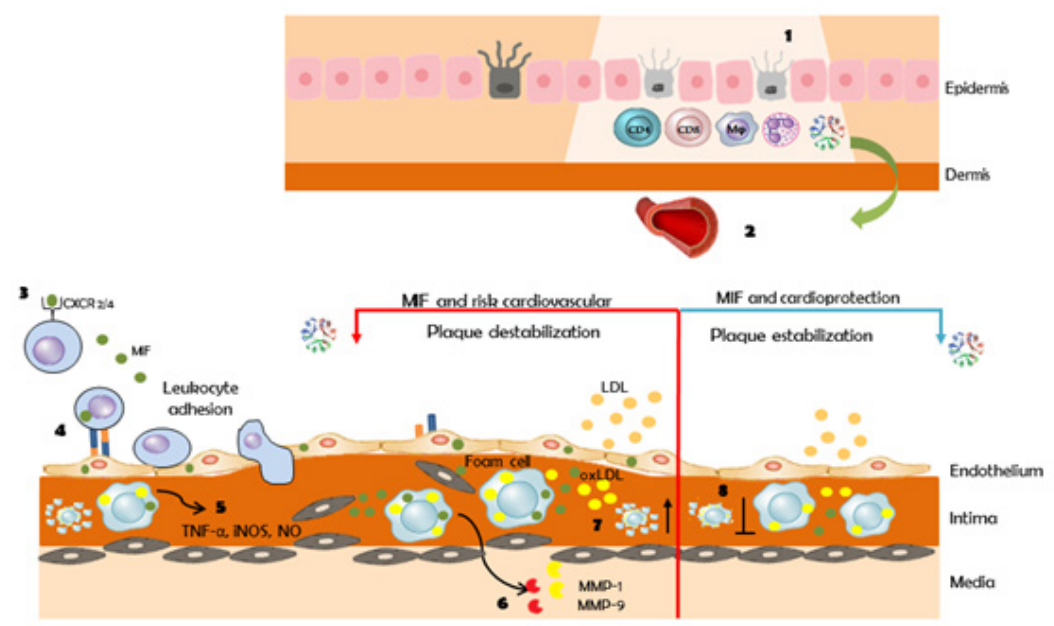

Figure 7.

\section{Discussion}

The pathogenesis of vitiligo remains unknown, however, many pathways of autoimmune, genetic, and environmental origin attempt to explain the loss of melanocytes in this disease. Genetic factors possess key roles in vitiligo as the disease is characterized by incomplete penetrance and multiple susceptibility loci [39]. In this respect, we observed that one-half of our patients had a positive family history of vitiligo, whereas only $8 \%$ of CS had this condition. This can be interpreted as that having a family member with vitiligo confers an 11.5-times greater risk of presenting the disease and is agree with that reported by Zhang et al. [40] who found approximately $50 \%$ heritability in vitiligo.

Studies performed on vitiligo skin biopsies have produced interesting data implicating activated dendritic cells, Th1, and Th17 cells in the effector responses targeting melanocytes [41]. Similar findings have been found in the serum of patients, reporting an increase in the proinflammatory Th1 and Th17 profile cytokines $[3,41,42]$.

MIF is a proinflammatory cytokine with a role not fully understood in vitiligo; however, it is known that its deletion or neutralization severely impairs the pro-inflammatory cytokine production, such as TNF- $\alpha$, IL-1 $\beta$, IL-6, IL-23, and IL-17, $[17,18,43]$ all of which are important in the pathogenesis of vitiligo. In the present study, we observed that MIF serum levels were higher in patients with vitiligo than in CS, in agreement with the only three reports, to our knowledge, published to date on this issue [4446]. However, unlike these authors, we did not find statistically significant differences in our population. Also, it was observed a significant positive relationship between MIF serum levels and the disease activity, as has been reported previously in Ma and Farag works, [44,46] but not by Serarslan [45] Regarding this, the pleiotropic immunologic and endocrine activities exerted by MIF suggest that its actions may be connected with complex signal transduction pathways. MIF has been associated with the pathogenesis of several chronic diseases, such as rheumatoid arthritis, [47] colitis, [48] types 1 and 2 Diabetes Mellitus, [49] chronic respiratory diseases [50,51], and cancer [52,53]. Moreover, MIF deficiency reduces chronic inflammation [54]. Since, vitiligo is an autoinflammatory disease and its activity is related to the appearance of new lesions or extension of the previous ones, grade +1 would indicate the presence of chronic and sustained inflammation. More studies are needed to find the mechanisms that regulate MIF's chronic secretion or function and to explain why elevated levels are not observed in stages of early activity.

The increase of pro-inflammatory cytokines such as IL- $1 \alpha$, IL-6, and TNF- $\alpha$ produce a chronic subclinical inflammatory state that would lead vitiligo patients to an increased predisposition for metabolic disorders, atherosclerosis, and cardiovascular damage $[5,6]$. Three papers have evaluated CR in patients with 
vitiligo: Karadag et al. [29] demonstrated that vitiligo patients have higher insulin resistance, elevated LDL levels, and decreased HDL levels; Shen and colleagues reported the presence of the HAIR-AN syndrome (hyperandrogenism, insulin resistance, and acanthosis nigricans) in an obese patient with vitiligo; [55] finally, RodríguezMartín et al. [56] concluded that patients with vitiligo seem to present fewer $\mathrm{CV}$ risks based in the findings that they show a better lipid profile, with higher levels of HDL, lower TG, and abdominal perimeter values than controls. Thus, the available evidence does not allow arriving at a firm conclusion about CR in this disease.

In this study, the CR was evaluated in patients with vitiligo through the lipid profile, the HOMA-IR, the atherogenic Index of Castelli, the usCRP, and the evaluation of the carotid IMT. The only lipid that presented a significant difference was the decrease in HDL, conferring to patients with vitiligo three times higher risk of having low HDL than subjects without the disease; this is important because low HDL levels are inversely correlated with the risk of CD [30]. In disagreement with Karadag et al. [29] we found no significant differences between groups concerning the insulin resistance and the elevated LDL levels, possibly because these authors did not pair patients and controls according to the BMI.

Based on the Framingham study, the Castelli Atherogenic Index and the usCRP are prognostic indicators of the atherosclerotic processes and identifies individuals with risk of heart disease [4957]. In this work, a higher average of the Castelli Atherogenic Index and the usCRP were observed in the subjects with vitiligo. Carotid atherosclerosis measured via IMT did not demonstrate significant differences between groups. Nonetheless, we noted that the average the IMT of the right carotid in subjects with vitiligo was $0.99 \mathrm{~mm}$, which alerts us to the fact that there is considerable damage at this level. Carotid IMT showed a positive relationship with the extent of the vitiligo. This could indicate that the greater the area of vitiligo, the higher the inflammatory state mediated by pro-inflammatory cytokines, leading to more significant damage to the vascular walls [58-60].

Considering that MIF has also been related to inflammation, atherosclerosis, and CDs, $[38,61,62]$ this study also evaluated its possible role as an early predictor of these diseases in vitiligo. There was a significant association between MIF levels and the HDL $<40 \mathrm{mg} / \mathrm{dL}$, insulin resistance, and the high-risk usCRP in patients with active vitiligo; this suggests a functional role of MIF as a CR factor in vitiligo patients. In this sense, it has been reported that genetic deletion of MIF blocked the development of glucose intolerance, insulin resistance, and the associated atherosclerotic disease in a murine model of induced obesity [63,64]. Also, MIF can control the transcription factor $\mathrm{C} / \mathrm{EBP} \beta$, a positive regulator of CRP $[63,65]$. Both insulin resistance and high-risk usCRP represent states of systemic inflammation and the values of MIF were found to be significantly different in these contexts only in the presence of the active state of vitiligo. The fundamental role of MIF as a central controller of systemic inflammation $[64,66]$ and the overall physiological importance of chronic inflammation in the pathogenesis of insulin resistance and associated atherosclerosis were supported in our work [63].

Another interesting find was a negative correlation between MIF with the insulin levels, HOMA-IR index, usCRP, VLDL-C, TG, left common carotid IMT, and left bulb IMT in patients with vitiligo regardless of the extent and the degree of disease activity. Verschuren et al. hypothesized that MIF could have a specific role modulating the inflammatory response without affecting directly common metabolic risk factors including plasma triglycerides, free fatty acids, VLDL, LDL, HDL, body weight, adipose mass, voluntary activity, and metabolic performance [63]. Thus, lowering chronic inflammation could be an effective strategy to block the development of metabolic diseases as well as CDs.

MIF has been also proposed by several authors as a biological marker of acute or severe conditions [67-71]. Studies that support CR associated with high levels of MIF are not an exception. For example, MIF expression is enhanced in acute coronary syndromes and it correlates with the extent of the cardiac necrosis, [23] as MIF levels were significantly higher in ST-segment elevated myocardial infarction patients with greater angiographic thrombus, [72] and patients with signs of plaque thrombosis showed significantly higher MIF levels [25]. Thus, those studies are consistent with the notion that circulating MIF levels are elevated promptly in response to an infarction in proportion to the mass of the affected myocardium. These works suggest that MIF may also have a cardioprotective effect during the acute disease stage.

MIF serum levels are typically found between 2 and $10 \mathrm{ng} / \mathrm{mL}$ in human plasma [73]. Notably, the mean value of MIF in our group was $3.02 \mathrm{ng} / \mathrm{mL}$. If we also consider that our patients had an average disease evolution of 13 years, it would seem possible to hypothesize that the negative correlation of MIF with cardiovascular study risk variables supports the cardioprotective role of this cytokine in acute events. Besides, different genetic variants in the MIF gene may be masking its effect on our study group, as reported by De la Cruz-Mosso et al. [14] in the population from Western Mexico.

Another relevant fact is that cardiomyocytes express and store MIF at a high level. During a myocardial infarction, the expression of MIF by circulating mononuclear cells remained unchanged, [74] indicating a cardiac source of circulating MIF in the acute phase of the pathology [24]. Thus, according to that observed in the skin of vitiligo patients, it is possible that the most important source of MIF concentrations could be the lesioned skin of the patients. Unfortunately, we were not able to correlate the optical densities of MIF tissue immunopositivity with serum levels of the protein because they were not equivalent samples. According to our results, the only study in the literature conducted by Ma et al. found 
that MIF mRNA levels were significantly higher in lesioned than in healthy skin [44].

In this research, the study group with vitiligo was stratified as a strategy that allows us to simplify and understand the most complex problems. In particular, in our study, we identify the set of parameters that could influence the relationship between MIF and vitiligo. However, the relatively small sample in each subgroup analysis could be an important limitation in the interpretation of our results. Greater efforts are required to demonstrate the causal relationship between MIF, vitiligo, and its possible complications.

\section{Conclusion}

In conclusion, our study supports the presence of a chronic inflammatory state in vitiligo that makes patients more vulnerable to cardiovascular disease and links to MIF as a master regulator of the immune response within the pathophysiology of the disease. Also, this work questions the role of MIF as a CR factor, since previous studies associate it more with acute CDs, leaving the doubt as to whether MIF is a predisposing factor or a protective-repairing mechanism of acute and severe heart injury. To our knowledge, the present study provides the first experimental evidence for the direct involvement of MIF in the evolution of CR variables in vitiligo patients.

\section{Declarations}

\section{Declaration of competing interests}

There is no conflict of interest of any of the authors with the results of this study.

\section{Funding}

This research received no specific grant from any funding agency in the public, commercial, or not-for-profit sectors.

\section{Ethical approval}

The ethics committee of The Dermatological Institute of Jalisco Dr. José Barba Rubio approved this study (No. 17-015).

\section{Guarantor}

LRL is the guarantor of this work.

\section{Contributorship}

LRL, ARL, and EGG conceived of the study. JATP, YMOG, PGF, AGO, NAA, JHB, ACMS, and EEPG performed the experiments. LRL, ARL, EGG, and JFMV wrote the manuscript. All authors analyzed and discussed the data. All authors read and approved the final manuscript.

\section{Acknowledgments}

We would like to thank the Pathology Laboratory of the Antiguo Hospital Civil of Guadalajara, Fray Antonio Alcalde for her assistance and guidance in this research

\section{References}

1. Guerra L, Dellambra E, Brescia S (2010) Vitiligo: pathogenetic hypotheses and targets for current therapies. Current drug metabolism 11(5): 451-67.

2. Huang CL, Nordlund JJ, Boissy R (2002) Vitiligo: a manifestation of apoptosis? Am J Clin Dermatol 3(5): 301-308.

3. Singh RK, Lee KM, Vujkovic-Cvijin I, Ucmak D, Farahnik B, et al. (2016) The role of IL-17 in vitiligo: A review. Autoimmun Rev 15(4): 397-404.

4. Laddha NC, Dwivedi M, Mansuri MS, Gani AR, Md Ansarullah, et al. (2013) Vitiligo: interplay between oxidative stress and immune system. Exp Dermatol 22(4): 245-250.

5. Aguilar-Salinas CA, Olaiz G, Valles V, Torres JM, Gómez Pérez FJ, Rull JA, et al. (2001) High prevalence of low HDL cholesterol concentrations and mixed hyperlipidemia in a Mexican nationwide survey. J Lipid Res 42(8): 1298-1307.

6. Bergqvist C, Ezzedine K (2020) Vitiligo: A Review. Dermatology 236(6): 571-592.

7. Feily A (2014) Vitiligo Extent Tensity Index (VETI) score: a new definition, assessment and treatment evaluation criteria in vitiligo. Dermatology practical \& conceptual 4(4): 81-84.

8. Birol A, Kisa U, Kurtipek GS, Kara F, Kocak M, et al. (2006) Increased tumor necrosis factor alpha (TNF-alpha) and interleukin 1 alpha (IL1-alpha) levels in the lesional skin of patients with nonsegmental vitiligo. Int J Dermatol 45(8): 992-993.

9. Taleb S (2016) Inflammation in atherosclerosis. Arch Cardiovasc Dis 109(12): 708-715.

10. Pant S, Deshmukh A, Gurumurthy GS, Pothineni NV, Watts TE, et al. (2014) Inflammation and atherosclerosis--revisited. J Cardiovasc Pharm T 19(2): 170-178.

11. Tillmann S, Bernhagen J, Noels H (2013) Arrest Functions of the MIF Ligand/Receptor Axes in Atherogenesis. Front Immunol 4: 115.

12. Stojanovic I, Saksida T, Nikolic I, Nicoletti F, Stosic-Grujicic S (2012) Macrophage migration inhibitory factor deficiency protects pancreatic islets from cytokine-induced apoptosis in vitro. Clin Exp Immunol 169(2): 156-163.

13. Llamas-Covarrubias MA, Valle Y, Bucala R, Navarro-Hernández RE, Palafox-Sánchez CA, et al. (2013) Macrophage migration inhibitory factor (MIF): genetic evidence for participation in early onset and early stage rheumatoid arthritis. Cytokine 61(3): 759-765.

14. De la Cruz-Mosso U, Bucala R, Palafox-Sánchez C, Parra-Rojas I, Padilla-Gutiérrez JR, et al. (2014) Macrophage migration inhibitory factor: Association of- 794 CATT5-8 and- 173 G> C polymorphisms with TNF- $\alpha$ in systemic lupus erythematosus. Hum Immunol 75(5): 433-439.

15. Morales-Zambrano R, Bautista-Herrera LA, De la Cruz-Mosso U, Villanueva-Quintero GD, Padilla-Gutiérrez JR, et al. (2014) Macrophage migration inhibitory factor (MIF) promoter polymorphisms (-794 CATT5-8 and-173 G>C): association with MIF and TNF $\alpha$ in psoriatic arthritis. Int J Clin Exp Med 7(9): 2605-2614.

16. Pazyar N, Feily A, Yaghoobi R (2013) Macrophage migration inhibitory factor as an incriminating agent in dermatological disorders. Indian J Dermatol 58(2): 157

17. Kim KW, Kim HR (2016) Macrophage migration inhibitory factor: a potential therapeutic target for rheumatoid arthritis. Korean J Intern Med 31(4): 634-642.

18. Stojanovic I, Cvjeticanin T, Lazaroski S, Stosić-Grujicić S, Miljković D (2009) Macrophage migration inhibitory factor stimulates interleukin-17 expression and production in lymph node cells. Immunology. 126(1): 74-83. 
19. Asare Y, Schmitt M, Bernhagen J (2013) The vascular biology of macrophage migration inhibitory factor (MIF). Expression and effects in inflammation, atherogenesis and angiogenesis. Thromb Haemostasis 109(3): 391-398.

20. Chen Z, Sakuma M, Zago AC, Zhang X, Shi C, et al. (2004) Evidence for a role of macrophage migration inhibitory factor in vascular disease. Arterioscl Throm Vas 24(4): 709-714.

21. Kleemann R, Hausser A, Geiger G, Mischke R, Burger-Kentischer A, et al. (2000) Intracellular action of the cytokine MIF to modulate AP-1 activity and the cell cycle through Jab1. Nature 408(6809): 211-216.

22. Pan J-H, Sukhova GK, Yang J-T, Wang B, Xie T, et al. (2004) Macrophage Migration Inhibitory Factor Deficiency Impairs Atherosclerosis in Low-Density Lipoprotein Receptor-Deficient Mice. Circulation. 109(25): 3149-3153

23. Muller II, Muller KA, Schonleber H, Karathanos A, Schneider M, et al. (2012) Macrophage migration inhibitory factor is enhanced in acute coronary syndromes and is associated with the inflammatory response. PloS one 7(6): e38376.

24. Deng XN, Wang XY, Yu HY, Shao-Min C, Xin-Ye X, et al. (2018) Admission macrophage migration inhibitory factor predicts long-term prognosis in patients with ST-elevation myocardial infarction. Eur Heart J 4(3): 208219.

25. Muller KA, Rath D, Schmid M, Schoenleber H, Gawaz M, et al. (2016) High Plasma Levels of Gremlin-1 and Macrophage Migration Inhibitory Factor, but Not Their Ratio, Indicate an Increased Risk for Acute Coronary Syndrome in Patients With Type 2 Diabetes Mellitus. Clin Cardiol 39(4): 201-206.

26. Boehncke WH, Boehncke S, Tobin AM (2011) The 'psoriatic march': a concept of how severe psoriasis may drive cardiovascular comorbidity. Exp Dermatol 20(4): 303-307.

27. Bonora E, Targher G, Alberiche M, Bonadonna RC, Saggiani F, et al. (2000) Homeostasis model assessment closely mirrors the glucose clamp technique in the assessment of insulin sensitivity: studies in subjects with various degrees of glucose tolerance and insulin sensitivity. Diabetes care 23(1): 57-63.

28. Ebrahim S, Papacosta O, Whincup P, Wannamethee G, Walker M, et al (1999) Carotid plaque, intima media thickness, cardiovascular risk factors, and prevalent cardiovascular disease in men and women: the British Regional Heart Study. Stroke 30(4): 841-850.

29. Karadag AS, Tutal E, Ertugrul DT (2011) Insulin resistance is increased in patients with vitiligo. Acta Derm-Venereol 91(5): 541-544.

30. Kopp HP, Kopp CW, Festa A, Krzyzanowska K, Kriwanek S, et al. (2003) Impact of weight loss on inflammatory proteins and their association with the insulin resistance syndrome in morbidly obese patients. Arterioscler Thromb Vasc Biol 23(6): 1042-1047.

31. National Cholesterol Education Program (NCEP) Expert Panel on Detection, Evaluation, and Treatment of High Blood Cholesterol in Adults (Adult Treatment Panel III) final report (2002) Third Report of the National Cholesterol Education Program (NCEP) Expert Panel on Detection, Evaluation, and Treatment of High Blood Cholesterol in Adults (Adult Treatment Panel III) final report. Circulation 106(25): 3143-3421.

32. Park SW, Kim SK, Cho Y-W, Kim DJ, Young-Duk S, et al. (2009) Insulin resistance and carotid atherosclerosis in patients with type 2 diabetes. Atherosclerosis 205(1): 309-313.

33. Parsad D, Gupta S (2008) Standard guidelines of care for vitiligo surgery. Indian J Dermatol Ve 74(Suppl): S37-S45.

34. Pearson TA, Mensah GA, Alexander RW, Anderson JL, Cannon RO, et al. (2003) Markers of inflammation and cardiovascular disease: application to clinical and public health practice: A statement for healthcare professionals from the Centers for Disease Control and Prevention and the American Heart Association. Circulation 107(3): 499-511.
35. Le Poole C, Boissy RE (1997) Vitiligo. Semin Cutan Med Surg 16(1): 3-14.

36. Schober A, Bernhagen J, Weber C (2008) Chemokine-like functions of MIF in atherosclerosis. J Mol Med (Berl) 86(7): 761-770.

37. Cheng Q, McKeown SJ, Santos L, Santiago FS, Khachigian LM, et al. (2010) Macrophage migration inhibitory factor increases leukocyte-endothelial interactions in human endothelial cells via promotion of expression of adhesion molecules. J Immunol 185(2): 1238-1247.

38. Tilstam PV, Qi D, Leng L, Young L, Bucala R, et al. (2017) MIF family cytokines in cardiovascular diseases and prospects for precision-based therapeutics. Expert Opin Ther Targets 21(7): 671-683.

39. Shen C, Gao J, Sheng Y, Dou J, Zhou F, et al. (2016) Genetic Susceptibility to Vitiligo: GWAS Approaches for Identifying Vitiligo Susceptibility Genes and Loci. Front Genet 7: 3.

40. Zhang XJ, Liu JB, Gui JP, Ming Li, Xiong QG, et al. (2004) Characteristics of genetic epidemiology and genetic models for vitiligo. J Am Acad Dermatol 51(3): 383-390.

41. Wang CQF, Cruz-Inigo AE, Fuentes-Duculan J, Moussai D, Gulati N, et al. (2011) Th17 Cells and Activated Dendritic Cells Are Increased in Vitiligo Lesions. PloS one 6(4): e18907.

42. Singh S, Singh U, Pandey SS (2012) Serum concentration of IL-6, IL-2, TNF- $\alpha$, and IFN $\gamma$ in Vitiligo patients. Indian J Dermatol 57(1): 12-14.

43. Reyes JL, Terrazas LI, Espinoza B, Cruz-Robles D, Soto V, et al. (2006) Macrophage migration inhibitory factor contributes to host defense against acute Trypanosoma cruzi infection. Infect Immun 74(6): 31703179.

44. Ma L, Xue HB, Guan XH, Shu CM, Zhang YJ, et al. (2013) Relationship of macrophage migration inhibitory factor levels in PBMCs, lesional skin and serum with disease severity and activity in vitiligo vulgaris. Braz J Med Biol Res 46(5): 460-464.

45. Serarslan G, Yonden Z, Sogut S, Savaş N, Celik E, et al. (2010) Macrophage migration inhibitory factor in patients with vitiligo and relationship between duration and clinical type of disease. Clin Exp Dermatol 35(5): 487-490.

46. Farag AGA, Hammam MA, Habib MS (2018) Macrophage migration inhibitory factor as an incriminating agent in vitiligo. An Bras Dermatol 93(2): 191-196.

47. Lue H, Kleemann R, Calandra T, Roger T, Bernhagen J, et al. (2002) Macrophage migration inhibitory factor (MIF): mechanisms of action and role in disease. Microbes and Infection 4: 449-460.

48. Jong Y, Abadia-Molina AC, Satoskar AR, Clarke K, Rietdijk ST, et al. (2001) The development of chronic colitis is dependent on the cytokine MIF. Nature Immunology 2(11): 1061-1066.

49. Sánchez-Zamora Y, Rodriguez-Sosa M (2014) The Role of MIF in Type 1 and Type 2 Diabetes Mellitus. Journal of Diabetes Research 2014: 804519.

50. Florez-Sampedro L, Soto-Gamez A, Poelarends G (2020) The role of MIF in chronic lung diseases: looking beyond inflammation. Am J Physiol Lung Cell Mol Physiol 318(6): L1183-L1197.

51. Günther S, Fagone P, Jalce G, Atanasov A, Guignabert C, et al. (2019) Role of MIF and D-DT in immune-inflammatory, autoimmune, and chronic respiratory diseases: from pathogenic factors to therapeutic targets. Drug Discovery Today 24(2): 428-439.

52. Lue H, Thiele M, Franz J, Dahl E, Speckgens S, et al. (2007) Macrophage migration inhibitory factor (MIF) promotes cell survival by activation of the Akt pathway and role for CSN5/JAB1 in the control of autocrine MIF activity. Oncogene 26(35): 5046-5059.

53. Tan L, Ye X, Zhou Y, Yu M, Fu Z, et al. (2014) Macrophage migration inhibitory factor is overexpressed in pancreatic cancer tissues and impairs insulin secretion function of $\beta$-cell. J Transl Med 12: 92. 
54. Verschuren L, Kooistra T, Bernhagen J, Voshol PJ, Margriet Ouwens D, et al. (2009) MIF Deficiency Reduces Chronic Inflammation in White Adipose Tissue and Impairs the Development of Insulin Resistance, Glucose Intolerance, and Associated Atherosclerotic Disease. Circulation Research 105(1): 99-107.

55. Shen Z, Hao F, Wei P (2009) HAIR-AN syndrome in a male adolescent with concomitant vitiligo. Arch Dermatol 145(4): 492-494.

56. Rodriguez-Martin M, de Paz NM, Mehtani P, Ferrer PC, Pestana Eliche M, et al. (2013) Patients with vitiligo present fewer cardiovascular risk factors: results from a case-control study. J Eur Acad Dermatol 27(1): 124-125.

57. Toledo-Corral CM, Ventura EE, Hodis HN, Weigensberg MJ, Lane CJ, et al. (2009) Persistence of the metabolic syndrome and its influence on carotid artery intima media thickness in overweight Latino children. Atherosclerosis 206(2): 594-598.

58. Tu CX, Gu JS, Lin XR (2003) Increased interleukin-6 and granulocyte-macrophage colony stimulating factor levels in the sera of patients with non-segmental vitiligo. J Dermatol Sci 31(1): 73-78.

59. Wang TJ, Gona P, Larson MG, Tofler GH, Levy D, et al. (2006) Multiple biomarkers for the prediction of first major cardiovascular events and death. N Engl J Med 355(25): 2631-2639.

60. Yang B, Li T, Wang J, Zhi G, Jin WS, et al. (2005) Insulin resistance and carotid atherosclerosis in 221 patients with potential hyperglycemia. Chinese medical sciences journal= Chung-kuo i hsueh k'o hsueh tsa chih 20(2): 108-111.

61. Noels H, Bernhagen J, Weber C (2009) Macrophage migration inhibitory factor: a noncanonical chemokine important in atherosclerosis. Trends Cardiovas Med 19(3): 76-86.

62. Kraemer S, Lue H, Zernecke A, Kapurniotu A, Andreetto E, et al. (2011) MIF-chemokine receptor interactions in atherogenesis are dependent on an N-loop-based 2-site binding mechanism. FASEB J 25(3): 894-906.

63. Verschuren L, Kooistra T, Bernhagen J, Voshol PJ, Ouwens DM, et al. (2009) MIF deficiency reduces chronic inflammation in white adipose tissue and impairs the development of insulin resistance, glucose intolerance, and associated atherosclerotic disease. Circ Res 105(1): 99-107.

64. Finucane OM, Reynolds CM, McGillicuddy FC, Harford KA, Morrison M, et al. (2014) Macrophage migration inhibitory factor deficiency amelio- rates high-fat diet induced insulin resistance in mice with reduced adipose inflammation and hepatic steatosis. PloS one 9(11): e113369.

65. Burger-Kentischer A, Gobel H, Kleemann R, Zernecke A, Bucala R, et al. (2006) Reduction of the aortic inflammatory response in spontaneous atherosclerosis by blockade of macrophage migration inhibitory factor (MIF). Atherosclerosis 184(1): 28-38.

66. Bernhagen J, Krohn R, Lue H, Julia L Gregory, Zernecke A, et al. (2007) MIF is a noncognate ligand of CXC chemokine receptors in inflammatory and atherogenic cell recruitment. Nat Med 13(5): 587.

67. Grieb G, Merk M, Bernhagen J, Bucala R (2010) Macrophage migration inhibitory factor (MIF): a promising biomarker. Drug news \& perspectives 23(4): 257-264

68. Østergaard C, Benfield T (2009) Macrophage migration inhibitory factor in cerebrospinal fluid from patients with central nervous system infection. Crit Care 13(3): R101.

69. Rahman SH, Menon KV, Holmfield JHM, McMahon MJ, Guillou JP (2007) Serum macrophage migration inhibitory factor is an early marker of pancreatic necrosis in acute pancreatitis. Ann Surg 245(2): 282289.

70. Otukesh H, Fereshtehnejad SM, Hoseini R, Hekmat S, Chalian H (2009) Urine macrophage migration inhibitory factor (MIF) in children with urinary tract infection: a possible predictor of acute pyelonephritis. Pediatr Nephrol 24(1): 105-111.

71. Bozza FA, Gomes RN, Japiassu AM, Soares M, Castro-Faria-Neto HC, et al. (2004) Macrophage migration inhibitory factor levels correlate with fatal outcome in sepsis. Shock 22(4): 309-313.

72. White DA, Fang L, Chan W, Morand EF, Kiriazis H, et al. (2013) Pro-Inflammatory Action of MIF in Acute Myocardial Infarction via Activation of Peripheral Blood Mononuclear Cells. PloS one 8(10): e76206.

73. Roger T, Schlapbach LJ, Schneider A, Weier M, Wellmann S, et al. (2017) Plasma Levels of Macrophage Migration Inhibitory Factor and d-Dopachrome Tautomerase Show a Highly Specific Profile in Early Life. Front Immunol 8: 26

74. Petrovsky N, Socha L, Silva D, Grossman AB, Metz C, et al. (2003) Macrophage migration inhibitory factor exhibits a pronounced circadian rhythm relevant to its role as a glucocorticoid counter-regulator. Immunol Cell Biol 81(2): 137-143. 